\title{
Supplementary Information: Single Electron Precision in the Measurement of Charge Distributions on Electrically Biased Graphene Nanotips Using Electron Holography
}

\author{
Leonardo Vicarelli,, ${ }^{1,2}$ Vadim Migunov, ${ }^{3,4, *}$ Sairam K. Malladi, ${ }^{1,5}$ \\ Henny W. Zandbergen, ${ }^{1}$ and Rafal E. Dunin-Borkowski ${ }^{3}$ \\ ${ }^{1}$ Kavli Institute of Nanoscience, Delft University of Technology, \\ Lorentzweg 1, 2628 CJ Delft, The Netherlands \\ ${ }^{2}$ Present address: NEST, CNR-Istituto Nanoscienze and Scuola Normale Superiore, \\ Piazza San Silvestro 12, I-56127 Pisa, Italy. \\ ${ }^{3}$ Ernst Ruska-Centre for Microscopy and Spectroscopy \\ with Electrons and Peter Grünberg Institute, \\ Forschungszentrum Jülich GmbH, 52425 Jülich, Germany \\ ${ }^{4}$ Central Facility for Electron Microscopy, \\ RWTH Aachen University, Ahornstraße 55, 52074 Aachen, Germany \\ ${ }^{5}$ Present address: Department of Materials Science and Metallurgical Engineering, \\ Indian Institute of Technology Hyderabad, \\ Yeddumailaram - 502205, Andhra Pradesh, India
}

(Dated: May 20, 2019) 


\section{EXPERIMENTAL DETAILS}

In situ experiments were performed in an FEI Titan G2 60-300 transmission electron microscope (TEM) operated at $300 \mathrm{kV}$. This microscope is equipped with two Möllenstedttype electrostatic biprisms [1]. The upper biprism, which is located above the selected area aperture plane, was used for off-axis electron holography.A simplified ray diagram and the experimental setup are shown schematically in Fig. S1. For each electron phase measurement, two sets of 10 object and 10 vacuum reference holograms were acquired with a charge-coupled device (CCD) camera using an exposure time of $6 \mathrm{~s}$. A representative electron hologram is shown in Fig. S2.
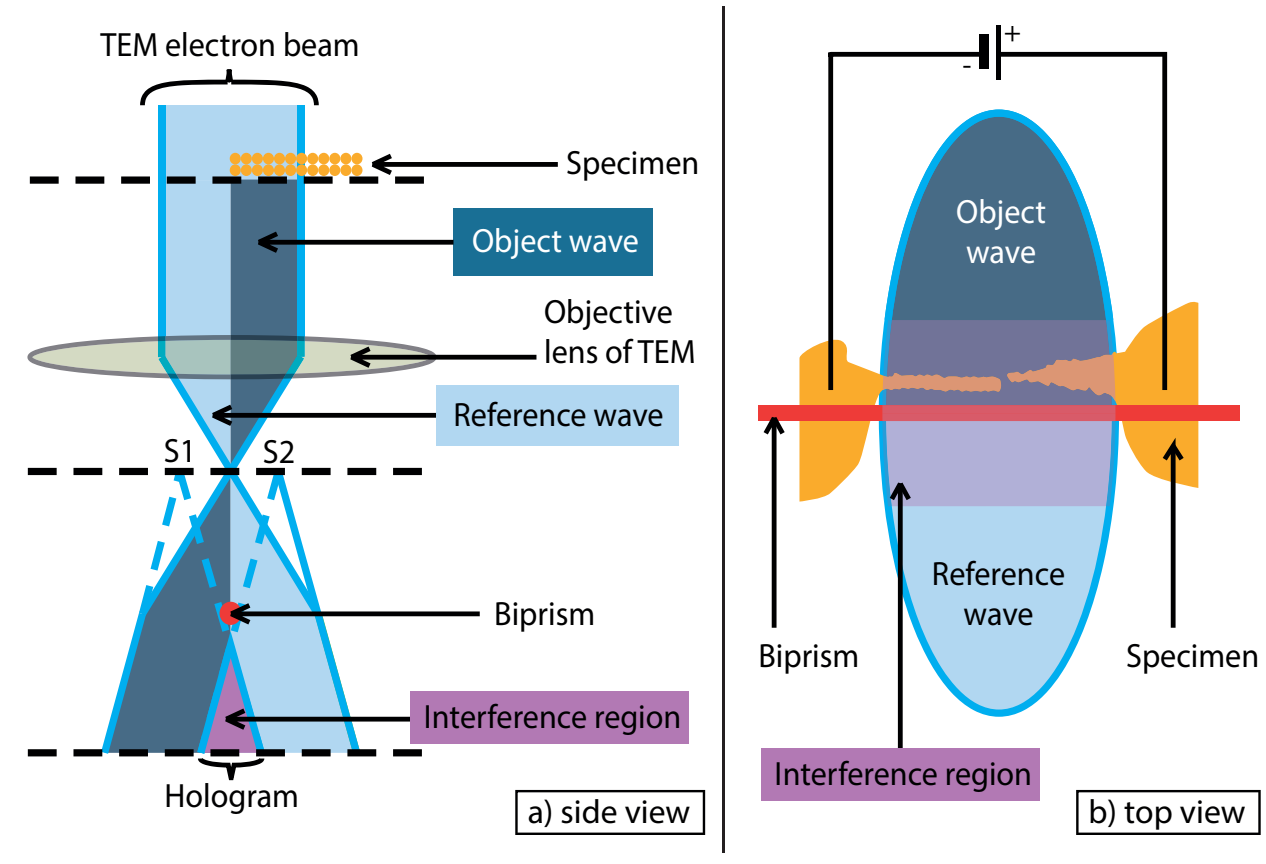

FIG. S1. Schematic diagrams of the setup for off-axis electron holography in the TEM. a) Simplified side view of the ray path. Virtual sources are denoted S1 and S2. b) Top view showing the geometry of the experiment in the specimen plane. The colours correspond to those used in panel (a). Panel (a) is adapted from Ref. [2].

Graphene nanoribbons were fabricated from exfoliated graphene and pre-patterned using standard electron beam lithography with a PMMA mask and an oxygen plasma etch. MEMS chips containing the graphene devices were placed in a home-built TEM specimen holder

\footnotetext{
*v.migunov@fz-juelich.de
} 


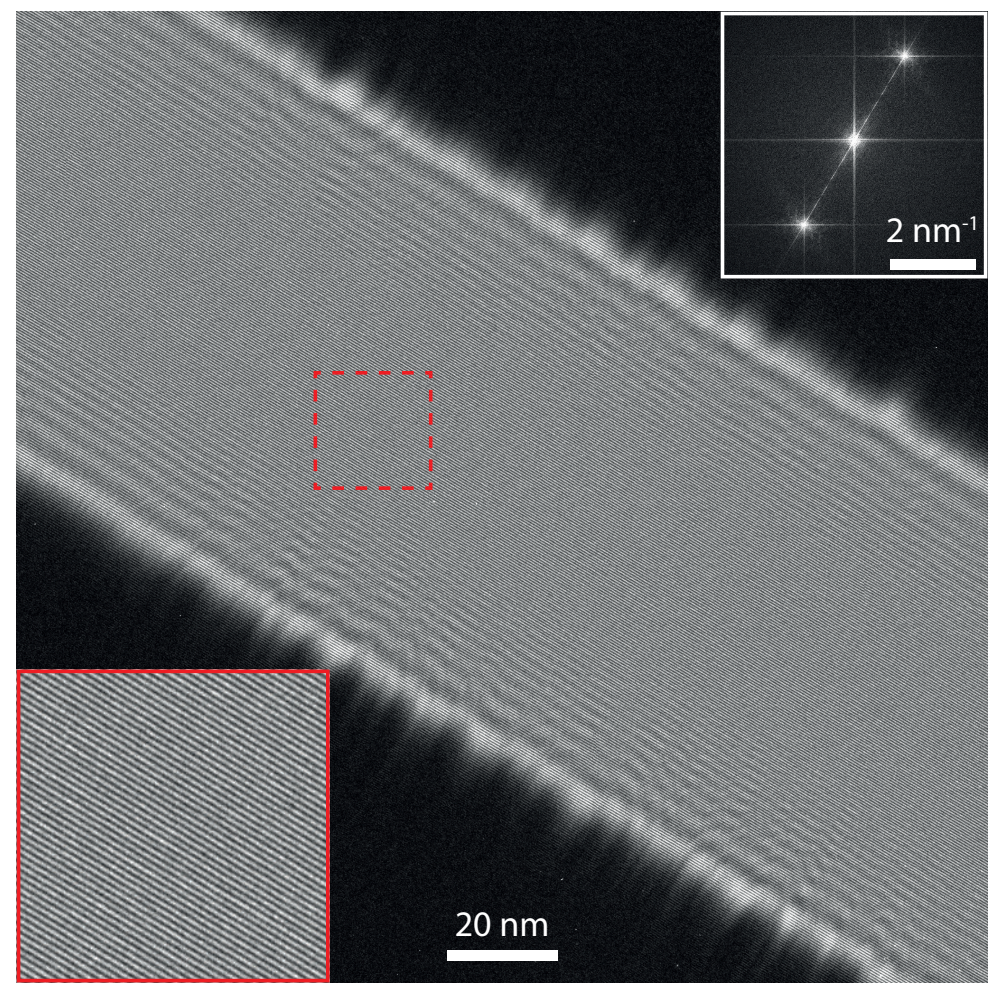

FIG. S2. Off-axis electron hologram recorded from device 3. This is one of 10 holograms, from which electron wavefunctions were reconstructed and averaged to calculate the phase shift shown in Fig. $2 \mathrm{~b}$ in the main text. The inset at the bottom left shows the region outlined with a red dashed square. The inset at the top right shows the central region of the Fourier transform of the hologram.

with 6 electrical contacts. Four of the contacts were used for in situ heating, while the remaining two were used for electrical biasing of the graphene nanogaps. The experiments were performed in vacuum $\left(\sim 10^{-7} \mathrm{mbar}\right)$, either at room temperature or at $300{ }^{\circ} \mathrm{C}$. Similar results were obtained at the two specimen temperatures.

Electrical breakdown of the nanoribbons (i.e., their decomposition into nanotips) was performed inside the TEM under high vacuum conditions. In order to induce breakdown, the voltage applied to each ribbon was ramped at a constant rate of $0.08 \mathrm{~V} / \mathrm{s}$, while monitoring the current passing through it. Figure S3 shows a representative current-voltage $(I-V)$ plot recorded during breakdown. An initial small drop in current (between 2.64 and 2.72 V) is followed by a rapid decrease in current at $2.72 \mathrm{~V}$ (faster than our temporal resolution of $\sim 10 \mathrm{~ms}$ ). Although the breakdown voltage varied from device to device, it always happened 


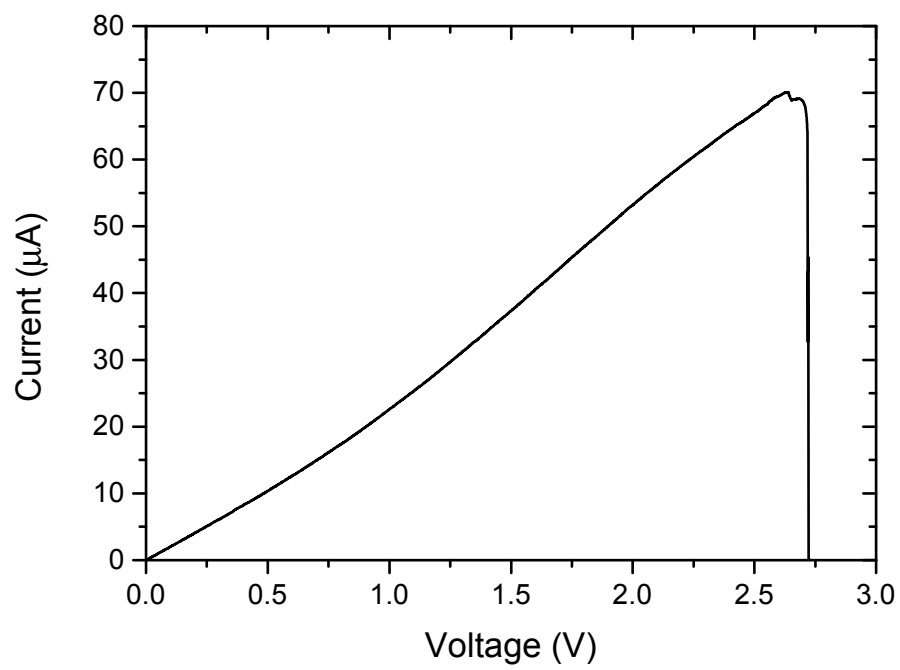

FIG. S3. Representative $I$ - $V$ curve recorded during the formation of a nanogap (i.e., during breakdown of a nanoribbon) as the voltage is swept at a constant rate of $0.08 \mathrm{~V} / \mathrm{s}$.

close to a critical current density of $5 \times 10^{8} \mathrm{~A} / \mathrm{cm}^{2}$.

EXPERIMENTS AND ANALYSES PERFORMED ON DIFFERENT DEVICES

Table S1 summarises results obtained from three graphene nanogap devices in this study. Device 2 was measured at three different bias voltages and showed a linear response of the total measured charge with applied voltage. Device 3 is described in the main text. 
TABLE S1. Projected charge densities measured in different graphene devices (average values on the two tips). Measurements were performed either at $300^{\circ} \mathrm{C}$ or at room temperature (RT).

*) Bias voltage refers here to the voltage difference between two phase images, which were subtracted from each other to remove the mean inner potential contribution to the signal from the results.

\begin{tabular}{lcccccr}
\hline \hline Device & $\begin{array}{c}\text { Temperature } \\
\text { number }\end{array}$ & $\begin{array}{c}\text { Gap } \\
\left({ }^{\circ} \mathrm{C}\right)\end{array}$ & $\begin{array}{c}\text { Thickness } \\
(\mathrm{nm})\end{array}$ & $\begin{array}{c}\text { Average } \\
\text { charge }\end{array}$ & $\begin{array}{c}\text { Bias } \\
\text { voltage* } \\
(\mathrm{e})\end{array}$ & $\begin{array}{r}\text { Capacitance } \\
(\mathrm{V})\end{array}$ \\
\hline 1 & 300 & 20 & $10-20$ & $16.3 \pm 1.1$ & 10 & 4 \\
2 & 300 & 58 & $6-7$ & $61.4 \pm 1.1$ & 10 & 1 \\
2 & 300 & 58 & $6-7$ & $30.4 \pm 1.1$ & 4.9 & 1 \\
2 & 300 & 58 & $6-7$ & $17.7 \pm 1.1$ & 2.8 & 1 \\
3 & $300 / \mathrm{RT}$ & 8 & $1-6$ & $17.6 \pm 1.1$ & 4 & 0.7 \\
\hline \hline
\end{tabular}

\section{PROJECTED CHARGE DENSITY}

The projected charges densities in the tips were measured using the expression $[5,6]$

$$
\sigma_{p}(x, y)=-\frac{\epsilon_{0}}{C_{E}} \nabla^{2} \phi(x, y)
$$

where $\sigma_{p}$ is the projected charge density, $\epsilon_{0}$ is the vacuum permittivity, $C_{E}$ is a constant that depends on the accelerating voltage of the microscope $\left(C_{E}=6.526 \mathrm{rad} \cdot \mathrm{V}^{-1} \cdot \mu \mathrm{m}^{-1}\right.$ at $300 \mathrm{kV}$ ) and $\phi$ is the recorded phase shift. In order to account for the fact that a discrete Laplacian needs to be calculated from a noisy image, the Laplace operator was convoluted by a Gaussian blurring filter, as suggested in Ref. [6].

Figures S4 and S5 and Fig. 2 in the main text show projected charge density distributions measured from the three devices. The wider devices (1 and 2; Figs S4 and S5) show a greater tendency to accumulate the induced charge around the edges of the graphene tips.

The noise level in the measurement and the ability to obtain single electron precision are assessed in Fig. S6. A line profile obtained from the vacuum region (Fig. S6c) shows that the noise level per pixel in this region does not exceed $10^{-3}$ electrons. (It should be noted that Gaussian filtering was applied when calculating the projected charge density). On the assumption that all pixels are independent (this is not strictly the case; the effect 

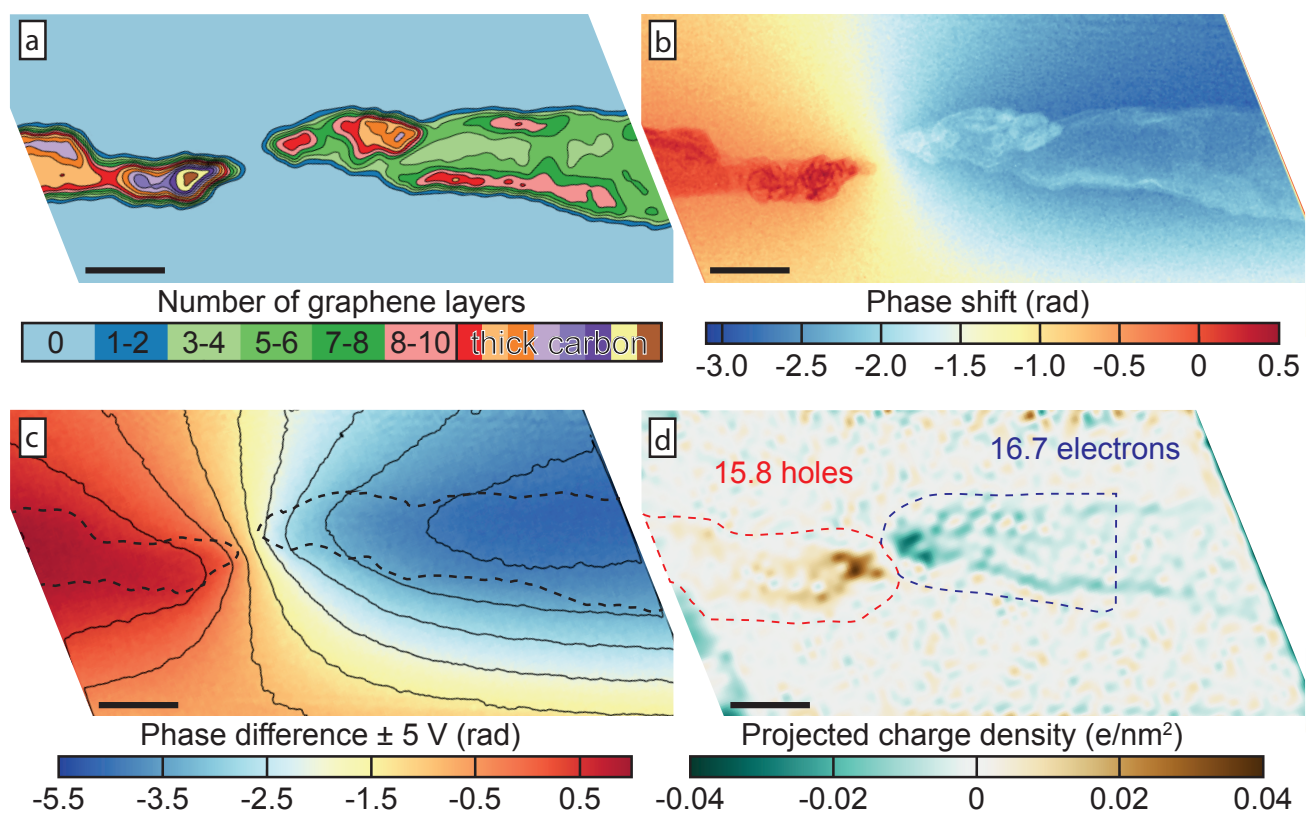

FIG. S4. Charge measurement in device 1 in Table S1. a) Electron phase shift at $0 \mathrm{~V}$ bias shown in units of the number of graphene layers (assuming a phase shift of $30 \mathrm{mrad}$ per layer at $300 \mathrm{kV}$ $[3,4])$. b) Electron phase shift measured at $5 \mathrm{~V}$ bias, showing the mean inner potential contribution to the phase of the graphene tips, in addition to a contribution arising from the applied bias voltage. c) Difference between electron optical phase images recorded at bias voltages of $\pm 5 \mathrm{~V}$. The black solid lines and the dashed lines mark equiphase contours separated by $0.6 \mathrm{rad}$ and the outlines of the tips, respectively. d) Laplacian of (c), showing the projected induced charge density distribution inside the graphene tips. The red and blue dashed lines show the areas in which the charge was integrated. Scale bar: $30 \mathrm{~nm}$.

of correlation is discussed below) and have an error of $\sigma_{x}=10^{-3}$ electrons, the integrated charge error $\sigma_{C}$ can be described according to the expression

$$
\sigma_{C}=\sqrt{\sum_{i}^{N}\left(\frac{\partial C}{\partial x_{i}} \sigma_{x_{i}}\right)^{2}}=\sqrt{\sum_{i}^{N} \sigma_{x}^{2}}=\sqrt{N} \sigma_{x}
$$

where the sum represents numerical integration over a chosen area that contains $N$ pixels with values $x_{i}$ and errors $\sigma_{x_{i}}$. The error depends on the size of the integration area. For typical integration regions of approximately 16000 pixels, the error in the integrated charge is approximately 0.13 unit charges. However, for correlated pixels the above equation needs to be corrected by a correlation coefficient, whose estimation is complicated by the presence of multiple sources of correlation (including Fourier filtering of the original holograms and 


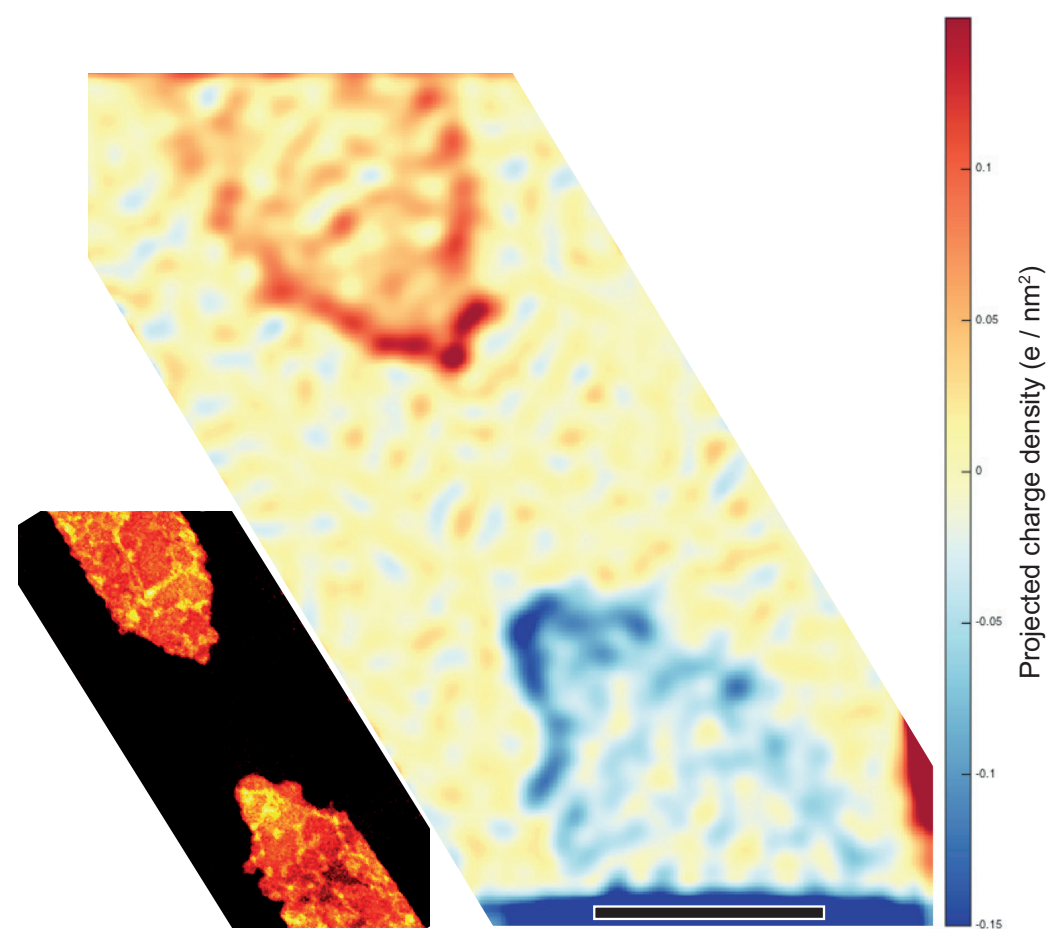

FIG. S5. Charge distribution in a graphene-based nanocapacitor (device 2 in Table S1). The inset at the lower left shows the mean inner potential contribution to the phase. The gap between the tips is $58 \mathrm{~nm}$ and their thickness is $\sim 6-7$ graphene monolayers. The areas at the top right and bottom left corners are outside the interference region and have been excluded as they contain only artefacts. Scale bar: $60 \mathrm{~nm}$.

the numerical Laplacian calculations performed on the phase). Here, we estimate the error in the integrated charge by evaluating the results of integration in vacuum and on the tips using different integration areas. The regions marked with black rectangles in Fig. S6a were used for charge integration. The area of each rectangle is $\sim 230 \mathrm{~nm}^{2}$. The measured total charge inside the rectangles does not exceed 0.25 electrons. Inside the tips, the integration was performed using 8 different areas. The results are shown in Fig. S6d. The variation of approximately 0.8 electrons, together with the noise estimates described above, confirms that the confidence level in the measurement of the total charge in each tip is better than a single electron. Considering our three error estimates of $0.13,0.25$, and 0.8 electrons associated with the vacuum noise in a single pixel, integration of the vacuum noise over a chosen area and the use of different integration areas inside the tips, respectively, we consider 0.8 electrons to be an upper limit for the error in our measurements and use this value in 

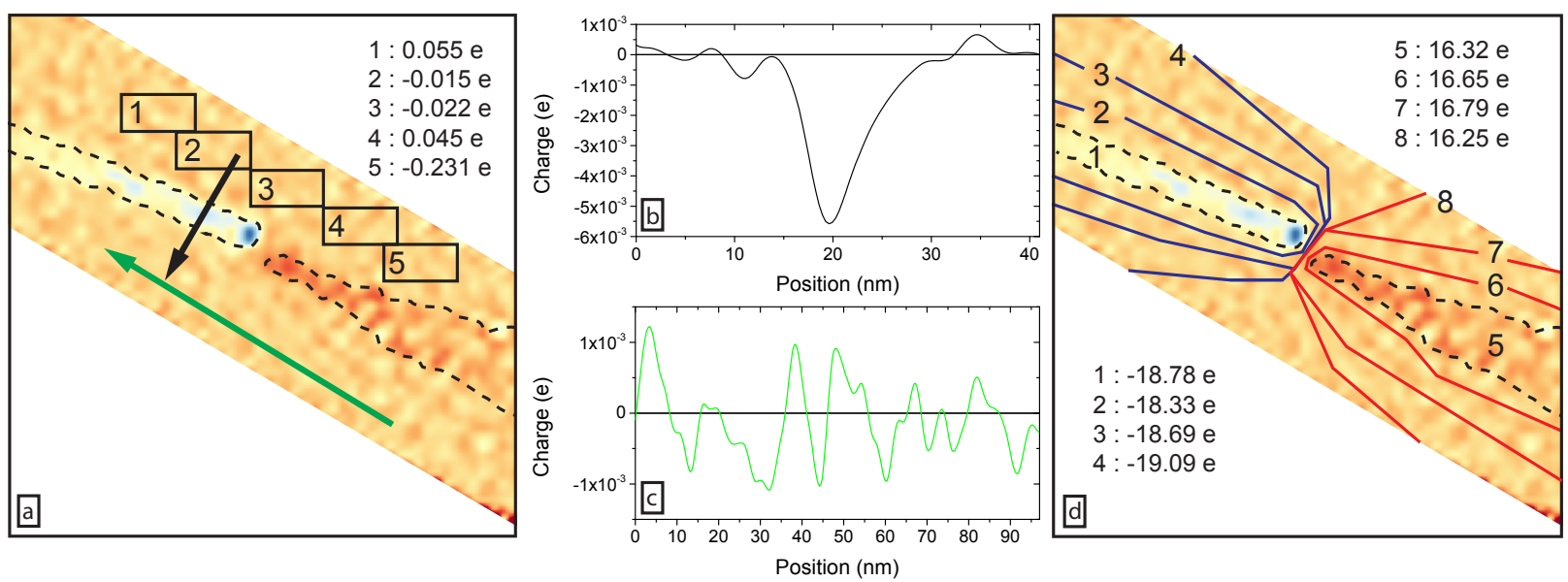

FIG. S6. Evaluation of noise in the charge density distribution measured in a graphene-based nanocapacitor (device 3 in Table S1). a) Charge density map. The black and green arrows show the positions and directions of the line profiles plotted in (b) and (c), respectively. The black rectangles show the reference areas used for integration of the total charge in vacuum, reflecting the noise level. The results of the integration are shown in the overlay. The area of each rectangle is $\sim 230 \mathrm{~nm}^{2}$. Each line profile is 1 pixel wide. The pixel size is $0.34 \mathrm{~nm}$. d) Charge density map with areas used for integration of the total charge in the tips outlined with blue and red lines. The results of the integration are shown in the overlay.

the present manuscript.

\section{COMPARISON OF EXPERIMENTAL RESULTS WITH SIMULATIONS}

Finite element method (FEM) simulations were performed classically using Comsol Multiphysics v5.2, without including quantum effects. The geometry of the device, including the SiN supporting membrane, was reproduced in the simulations.

The graphene tips were modeled as two-dimensional charged planes of zero thickness. In multilayer graphene, the interlayer spacing was set to $0.34 \mathrm{~nm}$, while keeping the individual layer thickness as zero. The choice of zero thickness for each graphene layer was intentional, as the simulation software would interpret a thicker layer as a combination of two distinct charged planes. The two-dimensional mesh that was used to describe graphene contained a total of 20206 triangular elements, as shown in Fig. S7a. A finer mesh of 6200 triangular elements was used to describe the part of the device within the field of view (Fig. S7b). 


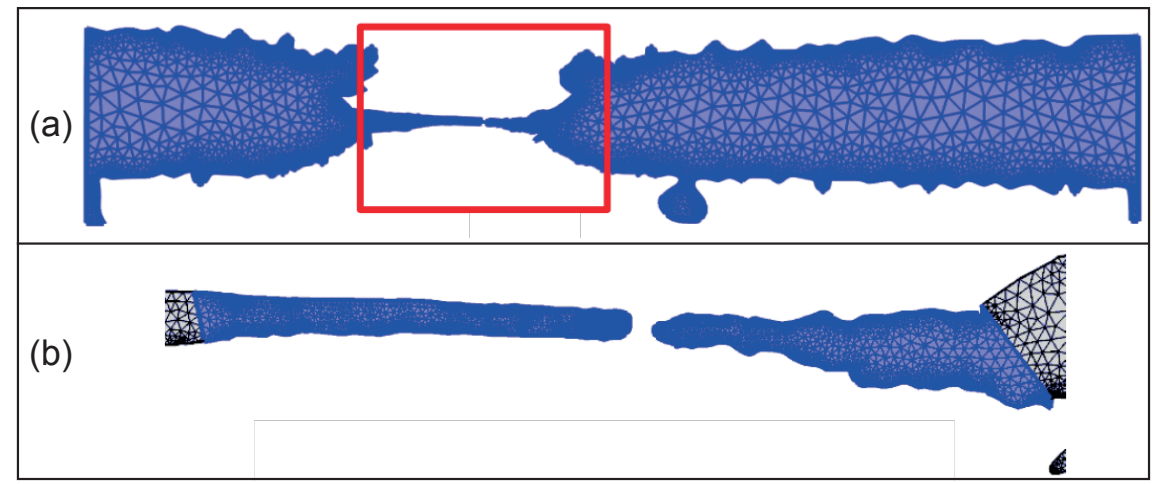

FIG. S7. Two-dimensional mesh used to describe a graphene nanogap device in finite element simulations. Panel (b) shows the part of region (a) that is marked by the red rectangle and corresponds to the field of view in the off-axis electron holography experiment.

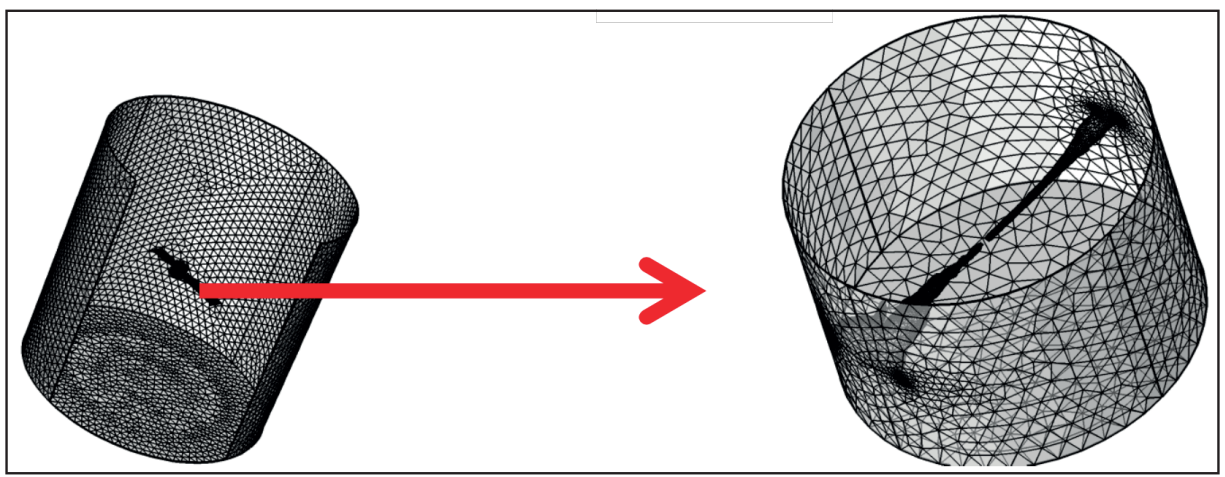

FIG. S8. Three-dimensional mesh used for finite element simulations of the volume (the cylinder has a diameter of $5 \mu \mathrm{m}$ and a height of $5 \mu \mathrm{m}$ ) used for calculations of the electrostatic potential around the graphene nanogap device. A sub-volume (a cylinder with a diameter of $300 \mathrm{~nm}$ and a height of $200 \mathrm{~nm}$ ) containing the experimental field of view is described by a finer mesh and is shown on the left.

The volume of the simulation was chosen to be a cylinder of height $5 \mu \mathrm{m}$ and diameter $5 \mu \mathrm{m}$, which was sufficient to contain most of the generated electrostatic potential. For higher precision, a sub-volume of cylindrical shape with a height of $200 \mathrm{~nm}$ and a diameter of $300 \mathrm{~nm}$, was defined using a finer mesh. The rough mesh includes 886393 tetrahedral elements, while the fine mesh in the sub-volume contains 381608 tetrahedral elements. They are both shown in Fig. S8.

The results of the simulations were exported to Matlab software for post-processing. The phase shift was obtained by integrating the electrostatic potential in a direction perpendicu- 
lar to the tip plane. The simulated electrostatic charge density was blurred using a Gaussian filter, similar to that applied to the experimental data. The phase shift corresponding to the perturbed reference wave was subtracted from the phase shift of the object wave, making use of the experimentally determined interference vector.

Figure S9 and Fig. 3 in the main text show comparisons between measured and simulated electron optical phase images for devices 2 and 3, respectively. An excellent match was obtained by taking into account the effect of the perturbed reference wave on the phase shift. By making use of both the simulations and the experiment results, the simulated perturbed reference wave (which is not known experimentally) could be subtracted from the experimentally recorded phase images.

TABLE S2. Total charge for device 3 in Table S1 obtained by FEM simulations.

\begin{tabular}{lcc}
\hline \hline $\begin{array}{l}\text { Number } \\
\text { of layers }\end{array}$ & Total charge \\
& Left tip & Right tip \\
\hline 1 & (electrons) & (holes) \\
2 & 16.2 & 13.1 \\
3 & 17.2 & 13.6 \\
4 & 17.7 & 14.2 \\
\hline \hline
\end{tabular}

\section{SUBTRACTION OF PERTURBED REFERENCE WAVE WITH SIMULATIONS}

The interference of an object electron wave with phase shift $\phi$ and a tilted reference wave with transverse wave vector $k_{0}$ in an idealised off-axis electron holography scheme can be described by the expression $[7]$

$$
I(\mathbf{r})=\left|\sqrt{N / 2} e^{-i k_{0} x}+\sqrt{N / 2} a(x) e^{i \phi(\mathbf{r})}\right|^{2}=\frac{N}{2}\left[1+a^{2}(\mathbf{r})+2 a(\mathbf{r}) \cos \left(k_{0} x+\phi(\mathbf{r})\right)\right],
$$

where $I(\mathbf{r})$ is the intensity at point $\mathrm{P}$ in the hologram (see Fig. S10), $N$ is the average number of counts in the vacuum region and $a(\mathbf{r})$ is the amplitude of the object wave. Fouriertransform-based reconstruction, which involves filtering and shifting the sideband at $k_{0}$ to the origin of reciprocal space and inverse Fourier transforming, is used to obtain the complex 

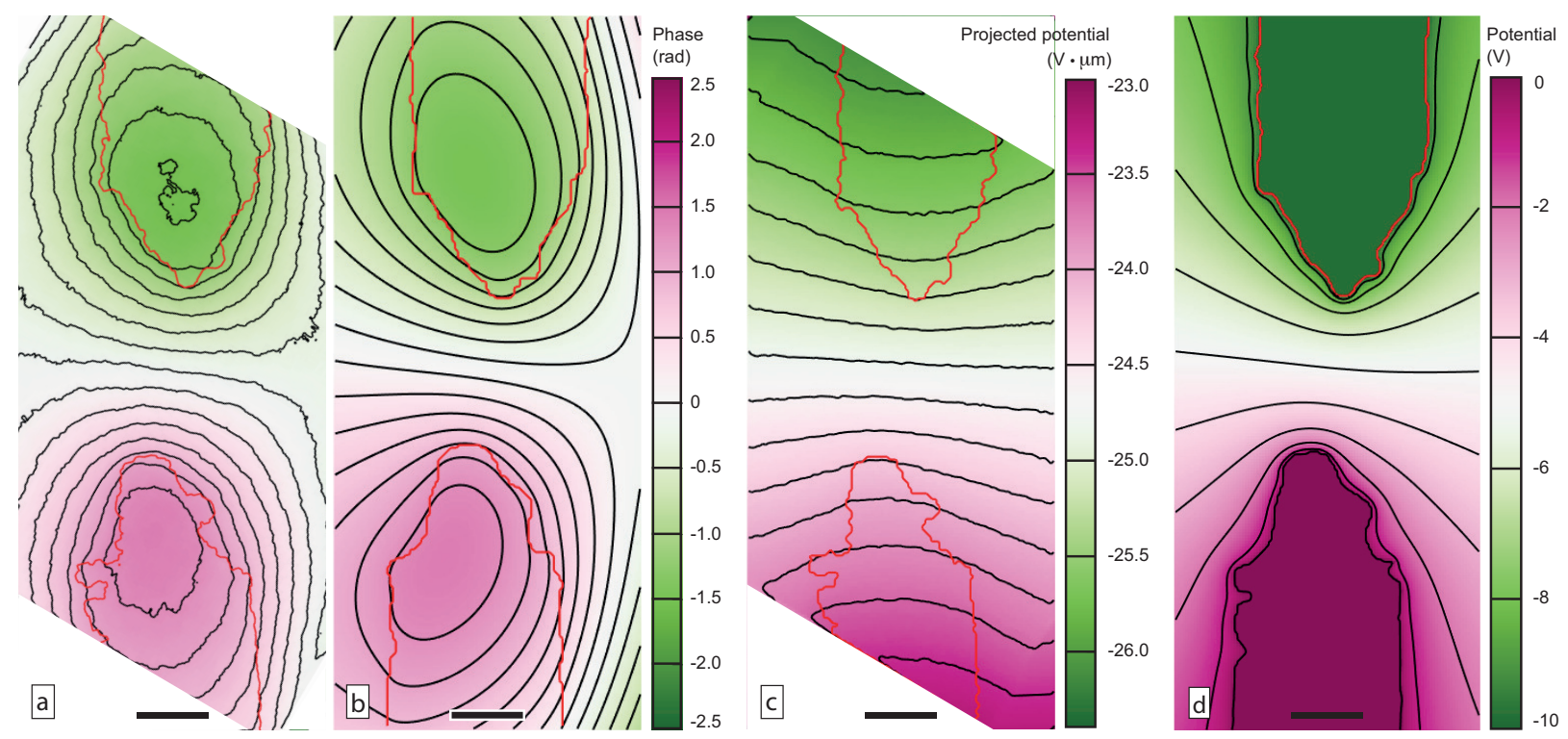

FIG. S9. Comparison between results obtained using electron holography and finite element simulations for device 2 in Table S1, with a bias voltage of $9.8 \mathrm{~V}$ applied between the tips, which are outlined in red. a, b) Experimental and simulated electron phase distributions. The contour spacing is $0.1 \mathrm{rad}$. The simulated phase shift includes the effect of the perturbed reference wave, for which the interference width and direction were determined experimentally. c) Projected electrostatic potential calculated from the experimental phase image after subtraction of the perturbed reference wave obtained from the simulation. The contour spacing is $0.2 \mathrm{~V} \cdot \mu \mathrm{m}$. d) In-plane distribution of electrostatic potential determined from the simulation. The contour spacing is $1 \mathrm{~V}$. Scale bar: $25 \mathrm{~nm}$.

object wavefunction. The influence on hologram formation of limited coherence, the details of the microscope imaging system, vignetting effects and other artefacts are omitted from Eq. 3. A comprehensive description of these effects can be found elsewhere [8-10].

If a long-range electromagnetic field affects the reference wave, then the phase of the reference wave $\phi(\mathbf{r}+\mathbf{D})$ will not be flat and Eq. 3 takes the form

$$
I(\mathbf{r})=\left|\sqrt{N / 2} e^{-i k_{0} x+i \phi(\mathbf{r}+\mathbf{D})}+\sqrt{N / 2} a(x) e^{i \phi(\mathbf{r})}\right|^{2}=\frac{N}{2}\left[1+a^{2}(\mathbf{r})+2 a(\mathbf{r}) \cos \left(k_{0} x+\phi_{T}(\mathbf{r})\right)\right],
$$

where $\mathbf{D}$ is the vector between two interfering points in the object plane [11]. This perturbed reference wave $(P R W)$ effect is illustrated schematically in Fig. S10. The reconstructed phase $\phi_{T}$ at point $\mathrm{P}$ is the difference between the phase at point $\mathrm{P}_{O}$ in the object wave and that 


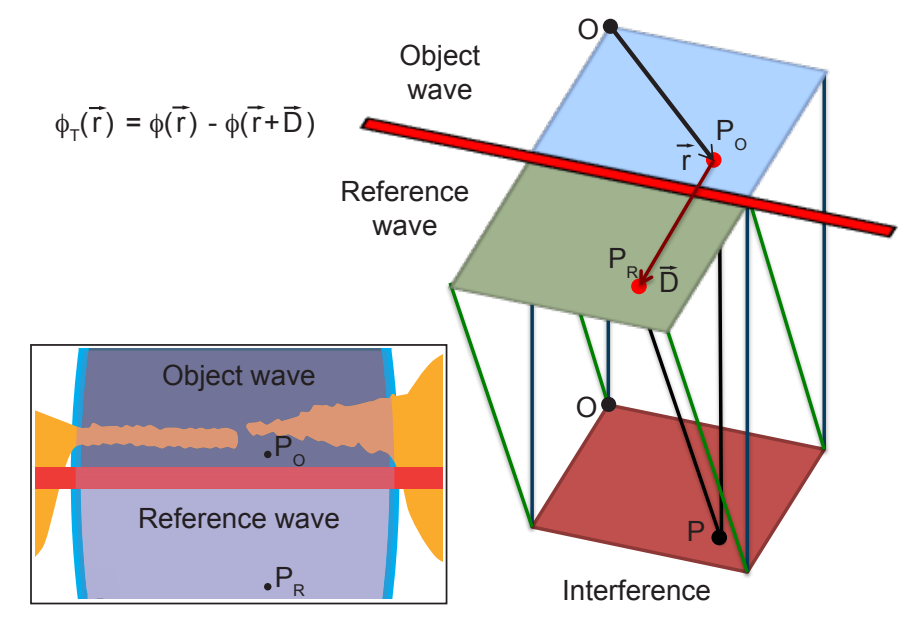

FIG. S10. Schematic diagram illustrating the effect of the perturbed reference wave. The object and reference electron waves (shown in light blue and green in the object plane) are overlapped by a biprism (red) to form an interference pattern in the image plane. The simplified ray paths from two interfering points $\mathrm{P}_{O}$ and $\mathrm{P}_{R}$ in the object plane are shown using black lines. The points are separated by an interference distance vector, which is the same for all points in the overlap (interference) region. The inset at the lower left corresponds to the central region of Fig. S1b and shows the experimental geometry of the object plane viewed in the direction of the electron beam.

at point $\mathrm{P}_{R}$ in the reference wave, according to the expression

$$
\phi_{T}(\mathbf{r})=\phi(\mathbf{r})-\phi(\mathbf{r}+\mathbf{D})
$$

For the graphene tips, we performed a correction for the effect of the PRW by using the phase shift obtained from the simulations. The phase shift was simulated for a larger field of view, including the object and reference wave regions. In order to obtain the corrected phase shift $(\phi(\mathbf{r})$ in Eq. 5), the simulated phase shift in the reference wave region $(\phi(\mathbf{r}+\mathbf{D}))$ was added to the experimentally measured phase shift $\left(\phi_{T}(\mathbf{r})\right)$. The interference distance vector $\mathbf{D}$, which is defined as the vector that connects any pair of points that interfere with each other, was determined as follows. The direction of $\mathbf{D}$ is perpendicular to the biprism and was measured from the holograms. Its magnitude is equal to the sum of the projected biprism width, measured from an image of the biprism (recorded in the absence of an applied voltage) and the overlap width measured from the original holograms. This correction provides an estimate of the real (unperturbed) phase shift and depends on the accuracy of the model that is used to describe the experiment. 


\section{FIELD EMISSION IN A GRAPHENE NANOGAP DEVICE}

A field emission experiment was performed at room temperature without electron beam illumination for device 1 (see Table S1) by sweeping the applied voltage between 0 and $22 \mathrm{~V}$. Figure S11 shows $I-V$ and Fowler-Nordheim $(\mathrm{F}-\mathrm{N})$ plots of the device recorded during the experiment. In the F-N plot, the onset of field emission can be identified at $\sim 15-16 \mathrm{~V}$. The field enhancement factor $\beta$ was calculated using the standard F-N relation for the slope of the linear part of the F-N plot (between 0.045 and 0.067 ) according to the expression

$$
\ln \left(\frac{I}{V^{2}}\right)=-B d \phi^{3 / 2} \frac{1}{\beta V}+\text { constant }
$$

where $B=6.83 \mathrm{eV}^{3 / 2} \times \mathrm{V} \times \mathrm{nm}^{-1}, d=20 \mathrm{~nm}$ is the gap between the graphene tips and $\phi=5 \mathrm{eV}$ is the work function of graphene. Using these parameters, the field enhancement factor $\beta$ takes a value of $22 \pm 7$. A low but non-zero conductance of the device was observed between 0 and $10 \mathrm{~V}$, most likely due to leakage in the thin SiN membrane.
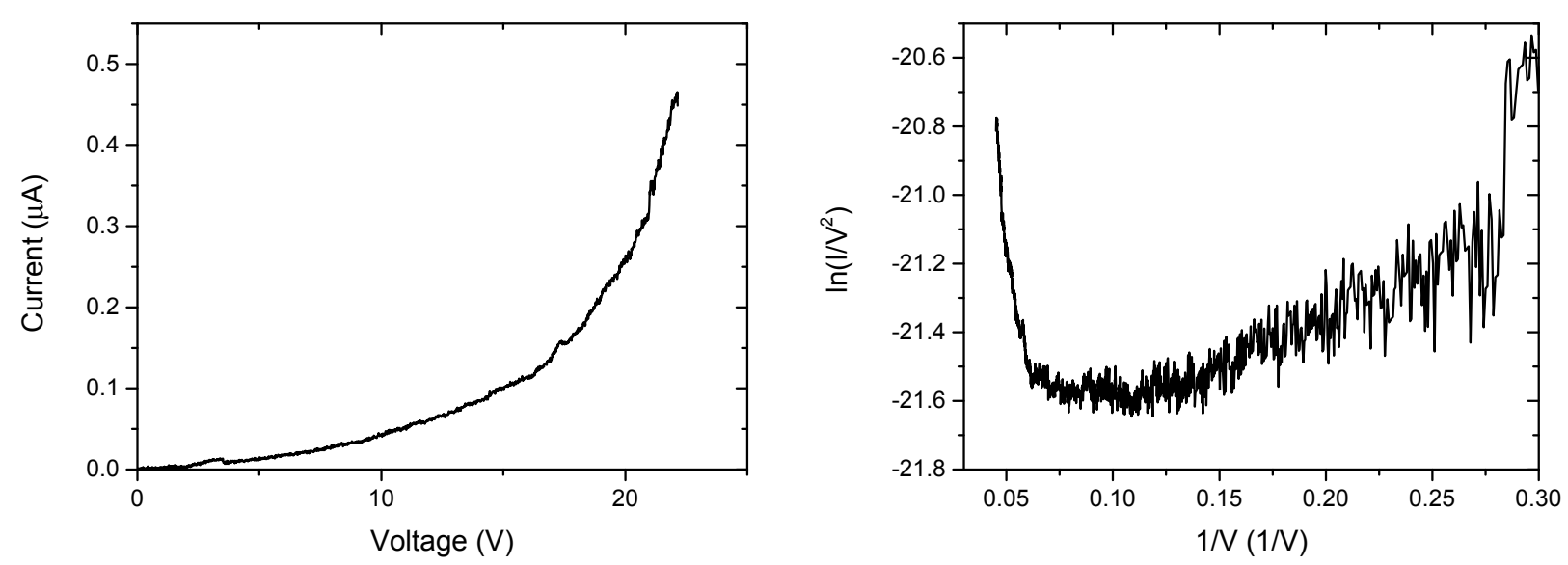

FIG. S11. Current-voltage (left) and Fowler-Nordheim (right) plots for device 1 (see Table S1) during field emission. 
[1] Möllenstedt, G.; Düker, H. Beobachtungen und Messungen an Biprisma-Interferenzen mit Elektronenwellen. Z. Physik 1956, 145, 377-397.

[2] Migunov, V. Elastic properties and electron transport in InAs nanowires. Dissertation, Universität Duisburg-Essen, Fakultät für Physik, Experimentalphysik, 2013.

[3] Ortolani, L.; Houdellier, F.; Monthioux, M.; Snoeck, E.; Morandi, V. Surface electrostatic potentials in carbon nanotubes and graphene membranes investigated with electron holography. Carbon 2011, 49, 1423-1429.

[4] Cooper, D.; Pan, C.-T.; Haigh, S. Atomic resolution electrostatic potential mapping of graphene sheets by off-axis electron holography. Journal of Applied Physics 2014, 115, 233709.

[5] Beleggia, M.; Kasama, T.; Dunin-Borkowski, R. E.; Hofmann, S.; Pozzi, G. Direct measurement of the charge distribution along a biased carbon nanotube bundle using electron holography. Applied Physics Letters 2011, 98, 243101.

[6] Beleggia, M.; Gontard, L. C.; Dunin-Borkowski, R. E. Local charge measurement using off-axis electron holography. J. Phys. D: Appl. Phys. 2016, 49, 294003.

[7] Migunov, V.; Dwyer, C.; Boothroyd, C. B.; Pozzi, G.; Dunin-Borkowski, R. E. Prospects for quantitative and time-resolved double and continuous exposure off-axis electron holography. Ultramicroscopy

[8] Lichte, H.; Lehmann, M. Electron holography-basics and applications. Rep. Prog. Phys. 2008, 71, 016102.

[9] Röder, F.; Lubk, A.; Wolf, D.; Niermann, T. Noise estimation for off-axis electron holography. Ultramicroscopy 2014, 144, 32-42.

[10] Chang, S. L. Y.; Dwyer, C.; Boothroyd, C. B.; Dunin-Borkowski, R. E. Optimising electron holography in the presence of partial coherence and instrument instabilities. Ultramicroscopy 2015, 151, 37-45.

[11] Matteucci, G.; Missiroli, G. F.; Pozzi, G. Electron holography of long-range electrostatic fields. Advances in Imaging and Electron Physics 2002, 122, 173-249. 